\title{
Reflets
}

Revue d'intervention sociale et communautaire

\section{Identité, pratiques et territoire}

\section{Marc Ferron}

Volume 17, numéro 2, automne 2011

URI : https://id.erudit.org/iderudit/1012135ar

DOI : https://doi.org/10.7202/1012135ar

Aller au sommaire du numéro

Éditeur(s)

Reflets, Revue d'intervention sociale et communautaire

ISSN

1203-4576 (imprimé)

1712-8498 (numérique)

Découvrir la revue

Citer cet article

Ferron, M. (2011). Identité, pratiques et territoire. Reflets, 17(2), 174-181.

https://doi.org/10.7202/1012135ar d'utilisation que vous pouvez consulter en ligne.

https://apropos.erudit.org/fr/usagers/politique-dutilisation/ 


\section{Identité, pratiques et territoire}

Marc Ferron, M.Scs., Mt.a., Hm.

Anthropologue, massothérapeute, homéopathe

Unité pédiatrique interculturelle, Centre de recherche de l'Hôpital Sainte-

Justine et Réseau de recherche en santé des populations du Québec Axe éthique

L'histoire et la santé des individus sont liées à leur environnement. D'abord, la famille est le premier environnement constituant le terreau du développement sociocognitif de l'enfant. Ses besoins, comblés ou non, construiront le sens de ses aspirations, et leur réalisation est dans l'ensemble liée à un environnement caractérisé par des potentialités variant selon les contextes. La qualité de la relation entre l'individu et son environnement est ainsi en grande partie déterminée par la prégnance du capital social, culturel et économique teintés par une symbolique originale à l'individu et à son milieu. C'est dans ce sens que sur le plan du capital social, identité individuelle et identité communautaire sont des concepts interdépendants où le développement de chacune des identités s'appuie sur les caractéristiques de l'autre. Ainsi, l'identité de l'individu et du groupe est le produit d'une collection de signes (Saussure, 1916) et de représentations sensibles aux influences des conventions historiques et des idéologies dominantes de l'environnement social (Gallissot, 1987).

Il y a donc deux vecteurs de l'identité : une identification fondatrice de l'appartenance sociale d'origine généalogique liée à la parenté, aussi constituée par les relations sociales de proximité (famille, travail, école, amis, quartier), et une identification de référence communautaire à caractère symbolique issue d'une projection collective actualisée, entre autres, dans le statut social et dans un partage d'idéologies communes (Gallissot, 1987). C'est pourquoi la ruralité, où la normalisation est très forte (Gallisot, 
2007) et constituée de réseaux sociaux denses, étendus et solidaires, est représentative d'une dynamique sociétale où l'identité des acteurs détermine l'actualisation des capitaux au sein de la communauté, laquelle influence l'activation de pratiques situées et de comportements d'adaptation caractéristiques du milieu (Giraud, 2001; Oriol, 1979; 1985; Alland, 1970; Leiban, 1973).

Incorporé dans une "matrice biologique " individuée et unique, l'humain vit au sein de groupes constituant des ensembles sociaux, géopolitiques, économiques et culturels reliés entre eux et médiatisant des représentations sur l'altérité et la normalité. Sur ce plan, les définitions de la maladie et de la santé de même que les stratégies pour la maintenir ou la rétablir sont diffusées et représentées dans des systèmes symboliques polysémiques connotés de croyances et de valeurs culturelles (Rivers, 1926). Entre individus et milieux, il y a la signification que les acteurs sociaux donnent à leurs relations, à leurs activités et à leurs comportements dans le but de satisfaire des besoins. Dans ce sens, les pratiques sociales, économiques et culturelles déterminent la position de la personne au sein de la société et sa relation par rapport à cet environnement. À ce sujet, les analyses anthropologiques indiquent que les notions de santé et de maladie sont déterminées par la place symbolique que le corps occupe au sein de l'espace social (Fassin, 1996) défini par la culture dominante, à la fois celle des institutions d'État, de la collectivité et de la famille. Sur ce plan, les rapports entre les domaines social, économique, culturel et biologique (Bourdieu, 1980; Black, 1988; Wilkinson, 1996; Fassin, 1996; Nguyen et Peschard, 2003, Bibeau, 2004) semblent tisser ensemble le canevas de la santé humaine où la maladie est conçue comme l'expression d'un déséquilibre entre le microcosme de l'individu et le macrocosme de l'ordre social (Lock, 2002). À ce sujet, la ruralité est un terrain propice pour expliquer le concept des "biologies locales ", lequel " éclaire en partie l'expérience incarnée, c'est-à-dire l'expérience des sensations physiques, notamment celle du bien-être, de la santé, de la maladie et qu'elles impliquent en réalité le corps matériel, mais que ce corps dépend lui-même de facteurs évolutifs, environnementaux, et autres » (Lock, 2005, p. 452). 
Située en Outaouais québécois, la Vallée de la Petite-Nation est un milieu rural représentatif d'une dynamique sociétale caractérisée par la densité et l'étendue des rapports familiaux et sociaux, une solidarité communautaire et une riche activité culturelle. Ici, le capital culturel et symbolique est issu de la culture familiale et communautaire conditionnée par l'histoire et la tradition diffusant un sens commun structuré autour de besoins communs. Cela soulève la question des relations entre individus, groupes et environnements modulés par l'influence du caractère symbolique des rapports d'appartenance avec le territoire (Oriol, 1979) exprimé par une relation à la fois sémantique, symbolique et pragmatique entre le corps et son milieu naturel (Oriol, 1979; Giraud, 2001). Ainsi, le mode de vie, les relations sociales et les usages du corps seraient intimement liés au type d'occupation, à la définition ainsi qu'aux pratiques et usages du territoire. Les comportements et les habitudes reflètent des modèles d'adaptation par la "relation symbolique et pratique " que les groupes humains entretiennent avec l'écosystème dans lequel ils s'investissent socialement, culturellement et économiquement (Oriol, 1979; Augé, 1994).

Un premier exemple exprimant la prégnance de la culture locale en rapport avec le territoire nous est donné par les habitudes, les représentations et les significations communes liées à la nature. Les habitants s'identifient fortement à la richesse de la faune, de la flore et la beauté des paysages dont ils tirent à la fois des revenus et un mode de vie. Mais implicitement, il y a la relation qu'ils entretiennent avec cette nature où l'existence se définit non pas seulement par le fait de vivre dans un environnement naturel, mais surtout d'être jusqu'à un certain point régie par les lois de la nature.Ainsi, dans plusieurs aspects de la vie quotidienne reliée au travail, aux loisirs, aux échanges et aux rituels sociaux, le rythme humain s'accorde à celui de la nature et le choix pour ce style de vie semble souvent s'exprimer par le corps, où plusieurs se représentent la santé comme l'état naturel du corps en équilibre avec la nature et son environnement social. Le milieu rural est cette nature, et les habitants ressentent profondément qu'ils font partie d'elle; ils ne peuvent ni s'en séparer, ni en nier l'importance, 
parce qu'ils lui doivent leur mode de vie, leur identité et leur santé. Un deuxième exemple offert par ce milieu, c'est qu'en plus des relations de travail et des transactions économiques, les relations sociales s'étendent à l'ensemble du territoire, sans apparence de barrières catégorielles, laissant ainsi la place à une communication de proximité qui participe à tisser un réseau social étendu. Les conversations spontanées à la terrasse du restaurant, dans les allées de l'épicerie ou devant le bureau de poste, avec un voisin, un ami, un parent ou un employé, meublent le quotidien. Dans ce contexte, la proximité des liens sociaux permet un dialogue où les aspects symboliques et identitaires se construisent au fil des échanges. Ce que les gens échangent d'abord en paroles qui se transposent ultérieurement en gestes d'échange et de solidarité est très significatif dans les représentations que les gens se font d'un mode de vie sain. Ainsi, une bonne partie de la vie rurale s'établit sur l'expérience intensive des rapports entre humains et entre humains et nature. C'est pour cela que nombre de ruraux ressentent vivement la rupture du tissu social et la contradiction inhérente au clivage homme-nature. Pour ceux-ci, il est impensable de vivre dans des conditions niant les principales références de leur univers symbolique, qu'on peut considérer comme une cosmogonie exprimant la culture d'une société qui vit du sens qui l'habite. Perçu comme fondamental, ce lien natureculture est représenté par l'équilibre entre homéostasie sociétale et santé humaine qui semble préservé par la dynamique des échanges sociaux et le contact avec la nature. Pour cela, plusieurs entretiennent une vision globale de leur santé, conscients qu'ils sont que le tout psychocorporel est construit par ses parties et qu'en tant qu'individus, ils sont une partie du tout social.

À cause de ces caractéristiques socioculturelles, les approches de santé s'adressant à une population particulière doivent démontrer une certaine réflexivité congruente avec les représentations que les gens se font de la santé et de la maladie, de la normalité et de l'altérité. À défaut de quoi, s'édifiera une barrière sémantique comme l'expression d'une opposition implicite ou explicite à un illogisme de sens menant à un échec thérapeutique. La médecine officielle avec sa connaissance rationnelle ne répond pas 
toujours à la signification que les gens donnent aux causes de leur maladie et dans un cadre de consultation où souffrances, douleurs, incompréhensions et peurs constituent la trame du discours, peu de patients se disent satisfaits du temps d'écoute qui leur est accordé. Pour cette raison beaucoup de gens recherchent des services thérapeutiques où le temps d'écoute permet l'expression d'une conception alternative de la santé dans laquelle est confirmé le lien entre nature et culture et où leur corps fait sens avec les significations qu'ils se représentent au sujet de leur maladie.

C'est ici que l'auteur se propose comme un acteur figurant au sein d'une dynamique sociétale où une pratique thérapeutique construite à son image s'est développée au fil du temps comme la réflexion à la fois de ses valeurs et de ses croyances en rapport avec celles de son milieu de vie. Citadin d'origine, à l'aube de sa carrière de massothérapeute utilisant une approche ostéopathique, et comme homéopathe il lui était devenu inconcevable de soigner le déséquilibre chez l'Autre tout en percevant être lui-même déséquilibré par la rupture du lien humain-nature et une certaine déconnexion sociale caractéristique du milieu urbain. Pour vivre ses valeurs à l'égard de la santé et affirmer son rôle en société, il devint évident qu'il lui fallait trouver un milieu représentatif de ses aspirations. Le milieu rural fut la réponse à cette quête de signification liée à une vie plus équilibrée centrée sur un rapprochement avec la nature et les gens.

D'emblée, trois aspects importants ont contribué à construire le sens de la thérapeutique telle qu'elle s'est définie au sein de la relation patient-thérapeute : la solidarité, la communication, les croyances et valeurs projetées sur la santé et sur le corps. D'office, on comprend qu'à un certain point, le thérapeute soit solidaire des valeurs que ses clients projettent sur leur corps et leur santé, mais cette solidarité va au-delà de la simple connivence des croyances parce qu'elle se transpose dans un support indéfectible au sens du cheminement thérapeutique signifié par le patient. Tout comme il n'apprécie guère se faire imposer une approche thérapeutique préconçue et inflexible, le thérapeute que je suis s'efforce de respecter les besoins, mais aussi les limites des croyances, des valeurs et des significations exprimées par ses patients. La relation 
d'aide ne s'établit pas par l'imposition de dogmes, mais bien par l'écoute de ce qui est signifiant pour la personne malade; ainsi, je ne cherche pas à déconstruire son univers en lui montrant "la voie ", mais plutôt à l'amener à découvrir si ses choix de vie, ses attitudes et ses comportements sont congruents avec ses besoins réels et sa santé. Sur ce plan, mon rôle thérapeutique est d'aider la personne à retrouver son équilibre fonctionnel en respectant le sens même des valeurs et des besoins exprimés, sauf si ceux-ci vont à l'encontre de son intégrité physique et psychique. Comme en milieu rural beaucoup de temps est consacré à la conversation, qui dans un certain sens participe à la définition de l'identité, le cadre thérapeutique réserve un temps à cet effet qui permet d'apprendre à connaître l'Autre et ses besoins et d'ouvrir la perspective des significations de la maladie et des moyens à envisager pour le retour de la normalité.

La confiance du patient envers le thérapeute se développe au sein de la communication où l'écoute de son discours suivie par une réponse informative du professionnel vient améliorer les connaissances du patient au sujet de son mal, contribuant ainsi à renforcer l'alliance thérapeutique. Dans le contexte socioculturel local de sous-scolarisation, la diffusion d'une information concrète, compréhensible et même rationnelle, mais surtout pertinente pour le malade et exprimée sur un ton incitant la confidence et dans un langage souvent métaphorique, contribue à l'éduquer et à le responsabiliser dans son cheminement tout en aidant à démystifier le rôle et les actions du thérapeute. Ici, la métaphorisation des phénomènes biologiques de la maladie et de la guérison vient bonifier un discours médical trop souvent distant des représentations populaires.

Le toucher par contre évoque un sens beaucoup plus intuitif permettant de construire un niveau de confiance qui ne relève pas uniquement d'un savoir positiviste. Cette approche convient bien à une clientèle pour qui le discours sur le corps, la santé et la douleur relève souvent d'une abstraction sémantique, mais où la qualité d'un toucher qui écoute tout en palpant, communique et infere en quelque sorte une reconnaissance du mal ouvrant la voie à une réponse curative. Aujourd'hui, tout comme avant, les 
services médicaux sont souvent difficiles d'accès et le thérapeute moderne tout comme le guérisseur de jadis représente souvent une voie plus rapide vers la réadaptation. De plus, parce que le souvenir des sages-femmes et des guérisseurs n'est pas si loin dans l'histoire locale, l'imaginaire populaire est encore imprégné par les représentations au sujet du caractère "magique " de ces ramancheurs, rebouteux et bone-setters (reformulé en Bonhomme Sept Heures). Et dans la symbolisation de la guérison, le toucher joue un rôle plus que significatif.

La communication s'actualise donc sur deux plans : dans l'écoute active des signifiants sémantiques et corporels où, en retour, le geste est informé d'un savoir par lequel le patient est touché tant par les mots que par les mains. Souvent, geste et parole sont liés dans la thérapeutique, car ils communiquent au malade ce qu'il n'aurait pu décoder lui-même. La qualité de la thérapie se base donc sur une communication favorisant un lâcher-prise face à la maladie, la souffrance et la douleur et c'est à partir de ce point que le patient est à même de retrouver son autonomie par la compréhension des signifiants de son mal. Disons simplement que c'est une manière de remettre le pouvoir entre les mains du patient afin qu'il se réapproprie son autonomie. Ainsi, l'utilisation d'une dialectique de solidarité par la communication, l'écoute et la reconnaissance identitaire liée au territoire et aux représentations symboliques (Oriol, 1983; Giraud, 2001) du corps et de la santé intégrées aux stratégies thérapeutiques et de promotion de la santé peuvent être considérées comme les éléments clés des processus de réadaptation agissant sur la qualité de vie et la santé des individus et populations.

Pour joindre Marc Ferron :

308-A Henri-Bourassa

Papineauville, Québec J0V 1R0

819-427-6229 (Bur)

819-308-0572 (tlcp) 


\section{Bibliographie}

AUGÉ, Marc (1994). Pour une anthropologie des mondes contemporains, Paris, Champs Flammarion Sciences, $195 \mathrm{p}$.

ALLAND, Alexander Jr. (1971). Adaptation in cultural evolution: An approach to medical anthropology. New York-London, Columbia University Press, 214 p.

BIBEAU, Gilles (2004). « Le capital social : vicissitudes d'un concept », Ruptures, Revue transdisciplinaire en santé, $\mathrm{Vol} 10, \mathrm{~N}^{\circ} 1$, p. 52-70.

BLACK, Douglas (1982). Inequalities in Health. The Black Report, London, Penguin Books, 240 p.

BOURDIEU, Pierre (1980). Le sens pratique, Paris, Éditions de Minuit, 500 p.

DE SAUSSURE, Ferdinand (1916). Cours de linguistique générale, [réédition 1979], Paris, Édition Payot, $520 \mathrm{p}$.

FASSIN, Didier (1996). La santé et le politique, l'espace politique de la santé : Essai de généalogie (Sociologie d'aujourd'hui), Paris, Presses universitaires de France, 324 p.

GALLISSOT, René (1987). "Sous l'identité, le procès de l'identification ", L'homme et la société, Vol. 83, No 1, p. 12-27.

GALLISSOT, René (2000). "Identité/Identification », dans R. Gallissot, M. Kilani et A. Rivera, L'imbroglio ethnique en quatorze mots clés, Lausanne, Éditions Payot, p. 133-143.

GIRAUD, Michel (2001). «Identité ", Pluriel Recherches, Cahier No.8, p. 39-51.

LIEBAN, Richard W. (1973). "Medical anthropology », dans J.J. Honigmann (dir.), Handbook of Social and Cultural Anthropology, Chicago, Rand MacNally, p. 1031-1072.

LOCK, Margaret (2002). « Medical Knowledge and Body Politics », dans Jeremy Macclancy (dir), Exotic No More : Anthropology on the Front Lines, Chicago, University Press, p. 190-208.

LOCK, Margaret (2005). «Anthropologie médicale : pistes d'avenir », dans F. Saillant et S. Genest, Anthropologie médicale. Ancrages locaux, défis globaux, Québec, Les presses de l'Université Laval, p. 439-467.

NGUYEN, Vinh-Kim, et Karine PESCHARD (2003). "Anthropology, inequality, and disease ", Annual Review of Anthropology, Montréal,Vol. 32, p. 447-474.

ORIOL, Michel (1979). "Identité produite, identité instituée, identité exprimée : confusion des théories de l'identité nationale et culturelle ", Cahiers internationaux de sociologie, Vol. 66, p. 1928.

ORIOL, Michel (1985). "L'ordre des identités », Revue européenne des migrations internationales, Vol.1, $\mathrm{N}^{\circ} 2$, p. 171-185.

RIVERS, William Halse Rivers (1926). Psychology and Ethnology, London, G. Elliott Smith, 352 p. http://books.google.com/books?hl=fr\&lr=\&id=uznwumfbBJEC\&oi=fnd\&pg=PR $9 \& d q=$ w.h . .r. + rivers + psychology + and + ethnology\&ots $=$ OnOASR xcOv\&sig $=w J W o A n i 2 \mathrm{crEn} 2 \mathrm{i} 46 \mathrm{Qk} 3 \mathrm{Q}$ EIjkofc $\# \mathrm{v}=$ onepage\&q\&f=false. Consulté sur internet février 2010.

WILKINSON, Richard G. (1996). Unhealthy societies. The afflictions of inequality, London et New York, Routledge, $272 \mathrm{p}$. 